Bull. Austral. Math. Soc.

Vol. 41 (1990) [487-494]

\title{
AN APPLICATION OF DIFFERENTIAL SUBORDINATIONS AND SOME CRITERIA FOR UNIVALENCY
}

\author{
Shigeyoshi OWa and Milutin Obradović
}

By using the method of differential subordinations, we derive, among other results, some criteria for univalency in the unit disc.

\section{INTRODUCTION AND PRELIMINARIES}

Let $A$ denote the class of functions $f(z)$ which are analytic in the unit disc $U=$ $\{z:|z|<1\}$ with $f(0)=f^{\prime}(0)-1=0$.

Let $f(z)$ be analytic in the unit disc $U$. Then the function $f(z)$ with $f(0)=0$ and $f^{\prime}(0) \neq 0$ is said to be starlike (univalent) if it satisfies

$$
\operatorname{Re}\left\{\frac{z f^{\prime}(z)}{f(z)}\right\}>0 \quad(z \in U)
$$

The function $f(z)$ with $f^{\prime}(0) \neq 0$ is said to be convex (univalent) if it satisfies

$$
\operatorname{Re}\left\{1+\frac{z f^{\prime \prime}(z)}{f^{\prime}(z)}\right\}>0 \quad(z \in U) .
$$

We note that $f(z)$ is convex in $U$ if and only if $z f^{\prime}(z)$ is starlike in $U$. Further, we denote by $S^{*}$ and $K$ the subclasses of $A$ consisting of functions $f(z)$ which are starlike and convex in $U$, respectively.

Let $f(z)$ and $F(z)$ be analytic in the unit disc $U$. Then the function $f(z)$ is said to be subordinate to $F(z)$, written $f(z) \prec F(z)$, if $F(z)$ is univalent in $U, f(0)=F(0)$ and $f(U) \subseteq F(U)$.

The general theory of differential subordinations was introduced by Miller and Mocanu [1]. The theory of first-order differential subordinations, with many interesting applications, especially in the theory of univalent functions, was considered by Miller and Mocanu [2]. Namely, if $\phi: \mathrm{C}^{2} \longrightarrow C$ (where $C$ is the complex plane) is analytic in a

Received 17 July 1989

This research was completed at the Department of Mathematics, Kinki University while the second author was visiting from the Department of Mathematics, Faculty of Technology and Metallurgy, University of Belgrade.

Copyright Clearance Centre, Inc. Serial-fee code: 0004-9729/90 SA2.00+0.00. 
domain $D$, if $h(z)$ is univalent in $U$, and if $p(z)$ is analytic in $U$ with $\left(p(z), z p^{\prime}(z)\right) \in D$ when $z \in U$, then $p(z)$ is said to satisfy a first-order differential subordination if

$$
\phi\left(p(z), z p^{\prime}(z)\right) \prec h(z) .
$$

The univalent function $q(z)$ is said to be a dominant of the differential subordination (1.3) if $p(z) \prec q(z)$ for all $p(z)$ satisfying (1.3). If $\bar{q}(z)$ is a dominant of (1.3) and $\widetilde{q}(z) \prec q(z)$ for all dominants of $(1.3)$, then $\widetilde{q}(z)$ is said to be the best dominant of the differential subordination (1.3).

By using the method of differential subordinations, we obtain a result which gives some criteria for univalency in the unit disc $U$. We note that we use methods similar to those used in [4].

The following lemmas are needed for the results in the next section.

Lemma 1. ([2]). Let $q(z)$ be univalent in the unit disc $U$, and let $\theta(w)$ and $\phi(w)$ be analytic in a domain $D$ containing $q(U)$, with $\phi(w) \neq 0$ when $w \in q(U)$. Set $Q(z)=z q^{\prime}(z) \phi(q(z)), h(z)=\theta(q(z))+Q(z)$, and suppose that

(i) $Q(z)$ is starlike in the unit disc $U$,

and

(ii) $\operatorname{Re}\left\{\left(z h^{\prime}(z)\right) /(Q(z))\right\}=\operatorname{Re}\left\{\left(\theta^{\prime}(q(z))\right) /(\phi(q(z)))+\left(z Q^{\prime}(z)\right) /(Q(z))\right\}$ $>0 \quad(z \in U)$.

If $p(z)$ is analytic in $U$, with $p(0)=q(0), p(U) \subseteq D$ and

$$
\theta(p(z))+z p^{\prime}(z) \phi(p(z)) \prec \theta(q(z))+z q^{\prime}(z) \phi(q(z))=h(z),
$$

then $p(z) \prec q(z)$, and $q(z)$ is the best dominant of the differential subordination (1.4).

Lemma 2. ([3]). If $f(z) \in A$ and

$$
\left|\arg \left(f^{\prime}(z)\right)\right|<\frac{\gamma_{0} \pi}{2}=0.968 \ldots \quad(z \in U),
$$

where $\gamma_{0}=0.6165 \ldots$ is the unique root of the equation

$$
2 \arctan (1-\gamma)+\pi(1-2 \gamma)=0,
$$

then $f(z) \in S^{*}$.

2. Differential SUBORDiNATION AND SOME CRITERIA FOR UNIVALENCY We first prove: 
TheOREM 1. Let $p(z)$ be analytic in $U$, with $p(0)=1$, and let $0<\lambda \leqslant 1$. If

$$
(1-\lambda) p(z)+\lambda z p^{\prime}(z) \prec\left(\frac{1+z}{1-z}\right)^{\gamma}\left(1-\lambda+\lambda \gamma \frac{2 z}{1-z^{2}}\right)=h(z),
$$

$0<\gamma \leqslant 1$, then

$$
p(z) \prec\left(\frac{1+z}{1-z}\right)^{\gamma}
$$

and this is the best dominant of (2.1).

Proof: We choose $q(z)=((1+z) /(1-z))^{\gamma}, 0<\gamma \leqslant 1, \phi(w)=\lambda$ and $\theta(w)=$ $(1-\lambda) w$ in Lemma 1 . Then the function $q(z)$ is convex in $U$ and $q(0)=1$. Further

$$
Q(z)=z q^{\prime}(z) \phi(q(z))=\lambda z q^{\prime}(z)
$$

is starlike, and for the function

$$
h(z)=\theta(q(z))+Q(z)=(1-\lambda) q(z)+\lambda z q^{\prime}(z)
$$

we have

$$
\operatorname{Re}\left\{\frac{z h^{\prime}(z)}{Q(z)}\right\}=\operatorname{Re}\left\{\frac{1-\lambda}{\lambda}+\frac{z Q^{\prime}(z)}{Q(z)}\right\}>0 \quad(z \in U) .
$$

Therefore the conditions (i) and (ii) in Lemma 1 are satisfied. By using Lemma 1 , we obtain that if $p(z)$ is analytic in the unit disc $U$ with $p(0)=1$ and

$$
(1-\lambda) p(z)+\lambda z p^{\prime}(z) \prec h(z),
$$

where $h(z)$ is defined in $(2.1)$, and $0<\lambda \leqslant 1$, then

$$
p(z) \prec q(z)=\left(\frac{1+z}{1-z}\right)^{\gamma}
$$

and this is the best dominant of the differential subordination (2.1).

In the case $\gamma=1$, Theorem 1 yields:

THEOREM 2. Let $p(z)$ be analytic in $U$ with $p(0)=1$, and $0<\lambda \leqslant 1$. If

$$
\begin{gathered}
\operatorname{Re}\left\{(1-\lambda) p(z)+\lambda z p^{\prime}(z)\right\}>-\frac{\lambda}{2} \quad(z \in U) \\
\operatorname{Re}\{p(z)\}>0 \quad(z \in U) .
\end{gathered}
$$

Proof: Taking $\gamma=1$ in Theorem 1 , we have $q(z)=(1+z) /(1-z)$ and

$$
h(z)=(1-\lambda) \frac{1+z}{1-z}+\lambda \frac{2 z}{(1-z)^{2}} \text {. }
$$


It follows from the above that

$$
\operatorname{Re}\left\{h\left(e^{i \phi}\right)\right\}=-\frac{\lambda}{2 \sin ^{2}(\phi / 2)} \leqslant-\frac{\lambda}{2} \quad(-\pi<\phi \leqslant \pi) .
$$

Also we have

$$
h(0)=1-\lambda \geqslant 0>-\frac{\lambda}{2} .
$$

Therefore (2.2) implies that the function $(1-\lambda) p(z)+\lambda z p^{\prime}(z)$ is subordinate to the function $h(z)$ defined by (2.3). By applying Theorem 1 , we conclude that

$$
p(z) \prec \frac{1+z}{1-z},
$$

that is, that $\operatorname{Re}\{p(z)\}>0$, which completes the proof of Theorem 2 .

Putting $f^{\prime}(z)$ with $f(z) \in A$ instead of $p(z)$ in Theorem 2, we have:

Corollary 1. Let $f(z) \in A$ and $0<\lambda \leqslant 1$. If

then

$$
\operatorname{Re}\left\{(1-\lambda) f^{\prime}(z)+\lambda z f^{\prime \prime}(z)\right\}>-\frac{\lambda}{2} \quad(z \in U),
$$

$$
\operatorname{Re}\left\{f^{\prime}(z)\right\}>0 \quad(z \in U) \text {. }
$$

If we take $\lambda=1$ and $z f^{\prime}(z) / f(z)$ or $1+z f^{\prime \prime}(z) / f^{\prime}(z)$ instead of $p(z)$ in Theorem 2, we obtain:

Corollary 2. If $f(z) \in A$ and

$$
\operatorname{Re}\left\{\frac{z f^{\prime}(z)}{f(z)}\left(1+\frac{z f^{\prime \prime}(z)}{f^{\prime}(z)}-\frac{z f^{\prime}(z)}{f(z)}\right)\right\}>-\frac{1}{2} \quad(z \in U)
$$

then $f(z) \in S^{*}$.

Corollary 3. If $f(z) \in A$ and

$$
\operatorname{Re}\left\{z^{2}\{f(z), z\}+\frac{1}{2}\left(1+\frac{z f^{\prime \prime}(z)}{f^{\prime}(z)}\right)^{2}\right\}>0 \quad(z \in U)
$$

where $\{f(z), z\}$ denotes the Schwarzian derivative defined by

$$
\{f(z), z\}=\left(\frac{f^{\prime \prime}(z)}{f^{\prime}(z)}\right)^{\prime}-\frac{1}{2}\left(\frac{f^{\prime \prime}(z)}{f^{\prime}(z)}\right)^{2}
$$

then $f(z) \in K$.

By a similar method to that used in Theorem 2, we may obtain the answer for the case $0<\gamma<1$, but this is more complicated than the case $\gamma=1$. Namely, we have: 
TheOREM 3. Let $p(z)$ be analytic in $U$ with $p(0)=1$, and let $0<\lambda \leqslant 1$ and $0<\gamma<1$. If

$$
\operatorname{Re}\left\{(1-\lambda) p(z)+\lambda z p^{\prime}(z)\right\}>C(\lambda, \gamma) \quad(z \in U)
$$

where

$$
\left\{\begin{aligned}
C(\lambda, \gamma) & =\frac{\lambda}{1+\gamma} t_{0}^{\gamma-1}\left(a t_{0}-\gamma\right) \sin (\gamma \pi / 2) \\
a & =\frac{1-\lambda}{\lambda} \cot (\gamma \pi / 2) \\
t_{0} & =\frac{a+\sqrt{a^{2}+1-\gamma^{2}}}{1+\gamma}
\end{aligned}\right.
$$

then

$$
|\arg (p(z))|<\frac{\gamma \pi}{2} \quad(z \in U)
$$

Proof: First we consider the function $h(z)$ defined in (2.1) for $0<\lambda \leqslant 1$ and $0<\gamma<1$. Noting that

$$
\begin{gathered}
h\left(e^{i \phi}\right)=\left(i \cot \frac{\phi}{2}\right)^{\gamma}\left(1-\lambda+i \frac{\lambda \gamma}{\sin \phi}\right) \quad(0<|\phi|<\pi) \\
i \cot \frac{\phi}{2}= \begin{cases}e^{i \pi / 2} \cot (\phi / 2) & (0<\phi<\pi), \\
-e^{-i \pi / 2} \cot (\phi / 2) & (-\pi<\phi<0),\end{cases}
\end{gathered}
$$

and

we obtain that

$$
\begin{aligned}
& \operatorname{Re}\left\{h\left(e^{i \phi}\right)\right\} \\
& \quad=\left( \pm \cot \frac{\phi}{2}\right)^{\gamma}\left((1-\lambda) \cos \left( \pm \frac{\gamma \pi}{2}\right)-\lambda \gamma \frac{\sin ( \pm \gamma \pi / 2)}{\sin \phi}\right), \quad(0<|\phi|<\pi)
\end{aligned}
$$

where we take " + " in the case $0<\phi<\pi$, and " - " in the case $-\pi<\phi<0$. It follows from (2.7) that $\operatorname{Re}\left\{h\left(e^{i \phi}\right)\right\}$ is an odd function. Thus we consider only the case

(2.8) $\operatorname{Re}\left\{h\left(e^{i \phi}\right)\right\}$

$$
=\left(\cot \frac{\phi}{2}\right)^{\gamma}\left((1-\lambda) \cos \left(\frac{\gamma \pi}{2}\right)-\lambda \gamma \frac{\sin (\gamma \pi / 2)}{\sin \phi}\right) \quad(0<\phi<\pi) .
$$

We shall show that

$$
\operatorname{Re}\left\{h\left(e^{i \phi}\right)\right\} \leqslant C(\lambda, \gamma) \quad(0<\phi<\pi)
$$


where $C(\lambda, \gamma)$ is defined by (2.5). Hence we put

$$
\cot \frac{\phi}{2}=t \quad(0<\phi<\pi) \text { and } a=\frac{1-\lambda}{\lambda} \cot \left(\frac{\gamma \pi}{2}\right) .
$$

Then (2.8) yields

$$
\operatorname{Re}\left\{h\left(e^{i \phi}\right)\right\}=g(t)=\frac{\lambda \gamma \sin (\gamma \pi / 2)}{2} g_{1}(t)
$$

where

$$
g_{1}(t)=\frac{2 a}{\gamma} t^{\gamma}-t^{\gamma-1}-t^{\gamma+1} \quad(0<t<+\infty) .
$$

It is easy to see that the function $g_{1}(t)$ defined by $(2.10)$ has the maximum value

at the point

$$
\begin{gathered}
g_{1}\left(t_{0}\right)=\frac{2 t_{0}^{\gamma-1}}{1+\gamma}\left(\frac{a}{\gamma} t_{0}-1\right) \\
t_{0}=\frac{a+\sqrt{a^{2}+1-\gamma^{2}}}{1+\gamma} .
\end{gathered}
$$

Therefore, from (2.9), (2.11) and the previous remark concerning $\phi$, we conclude that

$$
\operatorname{Re}\left\{h\left(e^{i \phi}\right)\right\} \leqslant C(\lambda, \gamma) \quad(-\pi<\phi \leqslant \pi),
$$

where, in the cases $\phi=0$ and $\phi=\pi$, we have

$$
\operatorname{Re}\left\{h\left(e^{i \phi}\right)\right\} \rightarrow-\infty .
$$

Also we may conclude that $C(\lambda, \gamma)$ is an increasing function of $t_{0}$, and that

$$
C(\lambda, \gamma) \rightarrow 1-\lambda
$$

when $t_{0} \rightarrow+\infty$ (equivalently, $\lambda \rightarrow 0$ ). This implies that

$$
\operatorname{Re}\left\{h\left(e^{i \phi}\right)\right\} \leqslant C(\lambda, \gamma)<1-\lambda=h(0) .
$$

Further, if $p(z)$ is analytic in $U$ with $p(0)=1$, and if

$$
\operatorname{Re}\left\{(1-\lambda) p(z)+\lambda z p^{\prime}(z)\right\}>C(\lambda, \gamma) \quad(z \in U),
$$

where $C(\lambda, \gamma)$ is defined by $(2.5)$, then, from the previous facts, we have that

$$
(1-\lambda) p(z)+\lambda z p^{\prime}(z) \prec h(z) \text {. }
$$

Finally, with the aid of Theorem 1, we obtain

and

$$
\begin{aligned}
& p(z) \prec\left(\frac{1+z}{1-z}\right)^{\gamma} \\
& |\arg (p(z))|<\frac{\gamma \pi}{2} .
\end{aligned}
$$


EXAMPLE 1. Letting $\gamma=1 / 2$ and $\lambda=1 / 2$ in Theorem 3 , we have $a=1$ and $t_{0}=(2+\sqrt{7}) / 3$. Then

$$
2 C(1 / 2,1 / 2)=\frac{\sqrt{6}(1+2 \sqrt{7})}{18 \sqrt{2+\sqrt{7}}}=0.3972 \ldots .
$$

Therefore we have that if

then

$$
\begin{gathered}
\operatorname{Re}\left\{p(z)+z p^{\prime}(z)\right\}>2 C(1 / 2,1 / 2)=0.3972 \ldots, \\
|\arg (p(z))|<\frac{\pi}{4} \quad(z \in U) .
\end{gathered}
$$

Combining Theorem 3 and Lemma 2, we obtain the following criterion for starlikeness.

Corollary 4. Let $f(z) \in A$, and let $0<\lambda \leqslant 1$ and $0<\gamma<1$. If

$$
\operatorname{Re}\left\{(1-\lambda) f^{\prime}(z)+\lambda z f^{\prime \prime}(z)\right\}>C\left(\lambda, \gamma_{0}\right) \quad(z \in U),
$$

where $\gamma_{0}$ is as in Lemma 2 and $C(\lambda, \gamma)$ is defined by (2.5), then $f(z) \in S^{*}$.

EXAMPle 2. For $\lambda=1$ in Theorem 3, we have that if $p(z)$ is analytic in $U$ with $p(0)=1$ and $0<\gamma<1$, then the following implication

where

$$
\begin{aligned}
& \operatorname{Re}\left\{z p^{\prime}(z)\right\}>C(1, \gamma) \Longrightarrow|\arg (p(z))|<\frac{\gamma \pi}{2} \\
& C(1, \gamma)=-\frac{\gamma}{1+\gamma}\left(\frac{1-\gamma}{1+\gamma}\right)^{(\gamma-1) / 2} \sin \left(\frac{\gamma \pi}{2}\right)
\end{aligned}
$$

is true. Therefore, from Corollary 4 , we obtain

$$
\operatorname{Re}\left\{z f^{\prime \prime}(z)\right\}>C\left(1, \gamma_{0}\right)=-0.414076 \ldots \Longrightarrow f(z) \in S^{*},
$$

where $\gamma_{0}$ is as in Lemma 2.

There remains the problem of finding the appropriate subset $E$ of the righthand halfplane such that $f(z) \in S^{*}$, whenever $f^{\prime}(z) \in E$ for all $z \in U$. For example, in Lemma 2, this type of problem was treated by Mocanu [3]. By using the result of Theorem 3, we may find other subsets which imply starlikeness, whenever $f^{\prime}(z)$ belong to them for all $z \in U$.

Theorem 4. Let $f(z) \in A$, and let $f^{\prime}(z)$ satisfy

$$
\left|\arg \left(f^{\prime}(z)\right)\right|<\frac{\gamma \pi}{2} \quad\left(\gamma_{0} \leqslant \gamma<1 ; z \in U\right),
$$


and

$$
\operatorname{Re}\left\{f^{\prime}(z)\right\}>2 C(1 / 2,1-\gamma) \quad(z \in Y)
$$

where $\gamma_{0}$ is as in Lemma 2 and $C(\lambda, \gamma)$ is defined by (2.5). Then $f(z) \in S^{*}$.

Proof: Letting $\lambda=1 / 2, p(z)=f(z) / z$ and $1-\gamma$ instead of $\gamma$ in Theorem 3, we have that (2.15) implies

$$
\left|\arg \frac{f(z)}{z}\right|<\frac{(1-\gamma) \pi}{2} \quad(z \in U)
$$

Therefore, using (2.14) and (2.16), we obtain

$$
\left|\arg \frac{z f^{\prime}(z)}{f(z)}\right| \leqslant\left|\arg \left(f^{\prime}(z)\right)\right|+\left|\arg \frac{f(z)}{z}\right|<\frac{\pi}{2},
$$

which shows that $f(z) \in S^{*}$.

\section{REFERENCES}

[1] S.S. Miller and P.T. Mocanu, 'Differential subordinations and univalent functions', Michigan Math. J. 28 (1981), 157-171.

[2] S.S. Miller and P.T. Mocanu, 'On some classes of first-order differential subordinations', Michigan Math. J. 32 (1985), 185-195.

[3] P.T. Mocanu, 'Some starlikeness conditions for analytic functions', Rev. Roumaine Math. Pures Appl. 33 (1988), 117-124.

[4] M. Obradović and S. Owa, 'An application of differential subordinations', Math. Nachr. 146 (1990) (to appear).

[5] M. Obradovic and S. Owa, 'An application of differential subordinations II', (submitted).

Department of Mathematics

Kinki University

Higashi-Osaka, Osaka 577

Japan
Department of Mathematics

Faculty of Technology and Metallurgy

University of Belgrade

4 Karnegieva Street

11000 Belgrade

Yugoslavia 